\title{
On the Stationary Convection of Thermohaline Problems of Veronis and Stern Types
}

\author{
Joginder S. Dhiman ${ }^{1 *}$, Praveen K. Sharma ${ }^{2}$, Poonam Sharma ${ }^{1}$ \\ ${ }^{1}$ Department of Mathematics, Himachal Pradesh University, Summerhill, Shimla, India \\ ${ }^{2}$ University Institute of Information Technology, Himachal Pradesh University, Summerhill, Shimla, India \\ E-mail:dhiman_jp@yahoo.com \\ Received July 28, 2010; revised September 20, 2010; accepted September 23, 2010
}

\begin{abstract}
The stability of thermohaline convection problems of Veronis and Stern types for stationary convection is studied for quite general nature of boundaries. It is shown by means of an appropriately chosen linear transformation, that in case of stationary convection the equations describing the eigenvalue problem for thermohaline convection problems are identical to equations describing the eigenvalue problem for classical Bénard convection problem. As a consequence, the values of the critical Rayleigh numbers for the onset of stationary convection in thermohaline convection problems are obtained. Also, necessary conditions for the validity of principle of exchange of stabilities for thermohaline convection problems are derived using variational principle.
\end{abstract}

Keywords: Thermohaline Convection, Stationary Convection, Eigenvalue Problem, Principle of Exchange of Stabilities, Rayleigh Number

\section{Introduction}

A problem in fluid mechanics involving the onset of convection has been of great interest for some time. Thermal convection occurs in nature in so many forms and over such a wide range of scales that it could be claimed with some justification that convection represents the most common fluid flow in the universe. The problem of onset of thermal instability in liquid layers heated from below originated from the experimental works of Bénard [1]. Stimulated by Bénard experiments, Lord Rayleigh [2] studied the Bénard problem mathematically for the first time, for the idealized case of both free boundaries and showed that the gravity dominated thermal instability in a liquid layer heated underside, depends upon the Rayleigh number. The theoretical treatments of convective problems usually invoked the so-called principle of exchange of stabilities (PES), which is demonstrated physically as convection occurring initially as a stationary convection. Alternatively, it can be stated as "the first unstable eigen values of the linearized system have imaginary part equal to zero".

A broader range of dynamical behaviour is observed in the convective instabilities that may occur when a fluid in a gravitational field contains two components of different diffusivities that affect the density, for example, temperature and solute. This phenomenon is known variously as thermohaline convection, double diffusive convection, or thermosolutal convection. Thermohaline convection, with its archetypal case of heat and salt, has been extensively studied in the recent past on account of its interesting complexities as well as its direct relevance in many problems of practical interest. Thermohaline convection has matured into a subject possessing fundamental departures from its classical counterpart, namely, thermal convection (single diffusive convection) and is of direct relevance to the fields of limnology, oceanography, astrophysics, etc. The various applications of the problem have aroused the interest of many research workers and this led to numerous research papers in various journals in the recent past. For a broad view of the subject one may be referred to Turner [3] and Brandt and Fernando [4].

Two fundamental configurations have been studied in the context of the thermohaline instability problem, one by Stern [5], wherein the temperature gradient is stabilizing and the concentration gradient is destabilizing and another by Veronis [6], wherein the temperature gradient is destabilizing and the concentration gradient is stabilizing. Further, one should also note the relationship of 
the Veronis's analysis to that of Stern's analysis. Veronis [6] has done the analysis of a situation that is gravitationally opposite to that of Stern's. Therefore, one can treat the two problems by considering the same configuration but with the assumption that gravity is positive downwards in one problem and positive upward in the other. It is important to note here that Veronis' as well as Stern's work are restricted to an idealized case of dynamically free boundaries.

Veronis also studied that when $R_{s}$ (salinity Rayleigh number) is sufficiently small (much less then $27 \pi^{4} / 4$, the value of critical Rayleigh number $R_{c}$ for ordinary convection with no solute present) the effect of the solute is to modify the results for ordinary convection by only a small amount. As $R_{s}$ is increased to the order of $R_{c}$, the value of $R$ (thermal Rayleigh number) at which the various types of instability can first occur also increases and as $R_{s}$ becomes very large the value of $R_{c}$ approaches asymptotic value. In other words, as $R_{s} \rightarrow \infty$, $R_{c} \rightarrow \tau^{-1} R_{s}$. Thus, the exact behaviour of the system as a function of $R_{s}$ depends on $\tau$ (the ratio of the diffusivities). Therefore, it becomes important to study this dependence for all combinations of boundaries. Further, it is evident that an analogous dependence for the case of Stern's type configuration may also hold.

Motivated by the above discussions regarding the stability and the structures of the thermohaline convection problems of Veronis and Stern types and their classical counterpart, the aim of the present paper is to study the stability of thermohaline convection problems for quite general nature of boundaries. It is shown here that upon using a linear transformation the equations describing the eigenvalue problem for stationary thermohaline convection problems become identical to equations describing the eigenvalue problem for stationary classical Bénard convection problem. As a consequence, the values of critical Rayleigh numbers for thermohaline convection problems of Veronis' and Stern's types for different combinations of rigid and dynamically free boundary conditions are obtained. Furthermore, necessary conditions for the validity of PES (in terms of $\tau^{-1} R_{s}$ and $\tau|R|$ - Laws) in Veronis and Stern types thermohaline convection problems are derived for sufficiently large values of Rayleigh numbers using variational principle.

\section{The Physical Configuration and the Basic Equations}

A viscous, quasi-incompressible (Boussinesq) fluid of infinite horizontal extension and finite vertical depth is statically confined between two horizontal boundaries $z=0$ and $z=d$ which are respectively maintained at uniform temperatures $T_{0}$ and $T_{1}$ and concentrations $C_{0}$ and $C_{1}$. We mathematically analyze the onset of convection in the system under the force field of gravity when the temperature and concentration make opposing contributions to the vertical density gradient. Following the usual steps of linear stability theory, the non-dimensional linearized perturbation equations governing the physical configuration described in the foregoing paragraph may be put in the forms [7];

$$
\begin{gathered}
\left(D^{2}-a^{2}\right)\left(D^{2}-a^{2}-\frac{p}{\sigma}\right) w \\
=R a^{2} \theta-R_{s} a^{2} \varphi \\
\left(D^{2}-a^{2}-p\right) \theta=-w \\
\left(D^{2}-a^{2}-\frac{p}{\tau}\right) \varphi=-\frac{w}{\tau}
\end{gathered}
$$

together with one of the boundary conditions;

$$
w=0=\theta=\varphi=D^{2} w \text { at } z=0 \text { and } z=1
$$

(both boundaries dynamically free)

or

$$
w=0=\theta=\varphi=D w \text { at } z=0 \text { and } z=1
$$

(both boundaries rigid)

or

$$
\begin{gathered}
w=0=\theta=\varphi=D w \text { at } z=0 \text { and } \\
w=0=\theta=\varphi=D^{2} w \text { at } z=1
\end{gathered}
$$

(lower boundary rigid and upper boundary dynamically free)

or

$$
\begin{aligned}
& w=0=\theta=\varphi=D w \text { at } z=1 \text { and } \\
& w=0=\theta=\varphi=D^{2} w \text { at } z=0
\end{aligned}
$$

(lower boundary dynamically free and upper boundary rigid)

In the above equations, $z$ is the real independent variable, $D=d / d z$ is the differentiation with respect to $z, a^{2}$ is the square of the wave number, $\sigma$ is the thermal Prandtl number, $\tau$ is the Lewis number, $R$ is the thermal Rayleigh number, $R_{s}$ is the concentration Rayleigh number, $p\left(=p_{r}+i p_{i}\right)$ is the complex growth rate and $w, \theta$ and $\varphi$ are the perturbations in the vertical velocity, temperature and concentration respectively and are complex valued functions of the vertical coordinate $z$ only.

\section{Mathematical Analysis}

The system of Equations (1)-(3) together with either of the boundary conditions (4) constitutes an eigenvalue problem for the complex growth rate $(p)$ for given values of the other parameters, namely, $\sigma, R, R_{s}$ and $\tau$ and a given state of the system is stable, neutral or unsta- 
ble according to whether $p_{r}$ is negative, zero or positive. Further, system of Equations (1)-(3) together with boundary conditions (4) describes the eigenvalue problem for

1) Veronis type thermohaline convection problem, if $R>0$ and $R_{s}>0$,

2) Stern type thermohaline convection problem, if $R<0$ and $R_{s}<0$

Furthermore, if $p_{r} \geq 0 \Rightarrow p_{i}=0 \forall a^{2}$, then for neutral stability, we have $p=0$. This is called PES.

\subsection{Critical Rayleigh Numbers}

When instability sets in as stationary convection, i.e., when PES is valid, Equations (1)-(3) for Veronis type thermohaline configuration becomes

$$
\begin{gathered}
\left(D^{2}-a^{2}\right)^{2} w=R a^{2} \theta-R_{s} a^{2} \varphi \\
\left(D^{2}-a^{2}\right) \theta=-w \\
\left(D^{2}-a^{2}\right) \varphi=-\frac{w}{\tau}
\end{gathered}
$$

together with any one of the boundary conditions (4).

Using the transformation $\chi=R \theta-R_{s} \varphi$, Equations (5)-(7) yield the following equations

$$
\begin{aligned}
& \left(D^{2}-a^{2}\right)^{2} w=a^{2} \chi \\
& \left(D^{2}-a^{2}\right) \chi=-\mathfrak{R} w
\end{aligned}
$$

and boundary conditions (4) become

$\chi=0$ at $z=0$ and $z=1$; and

either $D w=0$ or $D^{2} w=0$

$$
\text { at } z=0 \text { and } z=1
$$

Here, $\mathfrak{R}\left(=R-\frac{R_{s}}{\tau}\right)$ is termed as the effective Rayleigh number.

It is remarkable to note that Equations (8) and (9) are identical to classical Bénard equations [8], where $\mathfrak{R}$ plays the same role as that of $R$ (the thermal Rayleigh number) in Bénard convection problem. Therefore, the results already available for the thermal convection problem [8] can easily be translated into those for the thermohaline convection problem of Veronis type. Consequently, the values of critical Rayleigh number for Veronis type thermohaline convection problem for the following three cases of the boundary conditions can be obtained.

Following the analysis of [8], Equations (8)-(9) with the relevant boundary conditions from (10) yield the values of the critical Rayleigh numbers as;

Case I. When both boundaries are dynamically free.

$$
\Re_{c}=\frac{27 \pi^{4}}{4}=657.51
$$

this upon using the expression for $\mathfrak{R}_{c}$ implies that

$$
R_{c}=657.51+\frac{R_{s}}{\tau},
$$

the same value of the critical thermal Rayleigh number as obtained by Veronis [6] and Knobloch [9].

Case II. When both boundaries are rigid.

$$
R_{c}=1707.76+\frac{R_{s}}{\tau} .
$$

Case III. When one boundary is rigid and one is free.

$$
R_{c}=1100.65+\frac{R_{s}}{\tau}
$$

In the results (11)-(13), the values $657.51,1707.76$, 1100.65 are respectively the values of the critical thermal Rayleigh number for free-free, rigid-rigid, rigid-free boundary conditions respectively for the purely thermal problem [8].

The analogous values of critical Rayleigh number for Stern's type configuration can be easily obtained by replacing $R$ and $R_{s}$ by $-|R|$ and $-\left|R_{s}\right|$ respectively, in Equation (5) and emulating the analysis followed in Veronis type configuration. Therefore, we have

Case I. When both boundaries are dynamically free

$$
\left|R_{s}\right|_{c}=\tau(657.57+|R|) .
$$

Case II. When both boundaries are rigid

$$
\left|R_{s}\right|_{c}=\tau(1707.76+|R|) \text {. }
$$

Case III. When one boundary is rigid and one is free

$$
\left|R_{s}\right|_{c}=\left|R_{s}\right|_{c}=\tau(1100.65+|R|) \text {. }
$$

\section{2. $\tau^{-1} R_{s}$ and $\tau|R|$ - Laws}

From results (11)-(13), one can easily see that for sufficiently large values of $R_{s}$ (letting $\left.R_{s} \rightarrow \infty\right) R_{c} \rightarrow$ $\tau^{-1} R_{s}$.

Since the result holds for all the cases of boundary conditions, henceforth is referred to as $\tau^{-1} R_{s}$-law for Veronis type thermohaline convection problem. Also from (14-16), we can obtain an analogous law namely; $\tau|R|$-law for Stern's type configuration for sufficiently large values of $|R|$.

In the following sections, we shall validates the above stated laws using the minimum property of functional established by variational principle.

\section{A Variational Principle}

Letting

$$
F=\left(D^{2}-a^{2}\right)^{2} w
$$

and eliminating $\chi$ from Equations (8)-(9), we get 


$$
\left(D^{2}-a^{2}\right) F=-\mathfrak{R} a^{2} w
$$

and the boundary conditions corresponding to (10) as $w=0$ and $F=0$ at $z=0$ and $z=1$; and either

$$
D w=0 \text { or } D^{2} w=0 \text { at } z=0 \text { and } z=1
$$

depending on the nature of the bounding surfaces.

Multiplying Equation (18) by $F$ and integrating the resulting equation over the range of $z$, we get

$$
\begin{gathered}
\int_{0}^{1} F\left(D^{2}-a^{2}\right) F d z=-\mathfrak{R} a^{2} \int_{0}^{1} w F d z \\
=-\mathfrak{R} a^{2} \int_{0}^{1} w\left(D^{2}-a^{2}\right)^{2} w d z
\end{gathered}
$$

Integrating by parts the above equation a suitable number of times, using the relevant boundary conditions from (19), we get the expression for the functional as

$$
\begin{aligned}
\mathfrak{R} & =\frac{\int\left\{(D F)^{2}+a^{2}(F)^{2}\right\} d z}{a^{2} \int w F d z} \\
= & \frac{\int\left\{(D F)^{2}+a^{2}(F)^{2}\right\} d z}{a^{2} \int\left\{\left(D^{2}-a^{2}\right) w\right\}^{2} d z}=\frac{I_{1}}{a^{2} I_{2}},(s a y)
\end{aligned}
$$

where, $I_{1}$ and $I_{2}$ are positive definite integrals, and the limits of integration from the integral sign have been omitted for convenience in writing.

Upon using the value of $\mathfrak{R}$ and the positivity of $I_{1}$ and $I_{2}$, Equation (21) clearly implies that $R>\frac{R_{s}}{\tau}, \quad \forall a^{2}$.

Hence, for large values of $R_{s}$, we get

$$
R_{c} \geq \tau^{-1} R_{s} \text {. }
$$

It is remarkable to note here that the result (22) is uniformly valid for all cases of boundary conditions.

Using the variational method of Chandrasekhar's [8] for thermal convection problem, we have the stationary property of the functional $\mathfrak{R}$ given by expression (21) for all cases of boundary conditions given in (19) when the quantities on right hand side are evaluated in terms of true characteristic functions. Also the quantity on the right hand side of (21) attains its true minimum when $F$ belongs to $\mathfrak{R}_{c}$, i.e. the lowest characteristic value of $\mathfrak{R}$, namely $\mathfrak{R}_{c}$, is indeed a true minimum, i.e.

$$
\begin{aligned}
\mathfrak{R}_{c}(= & \left.R_{c}-\frac{R_{s}}{\tau}\right) \leq \frac{\int\left\{(D F)^{2}+a^{2}(F)^{2}\right\} d z}{a^{2} \int w F d z} \\
= & \frac{\int\left\{(D F)^{2}+a^{2}(F)^{2}\right\} d z}{a^{2} \int\left\{\left(D^{2}-a^{2}\right) w\right\}^{2} d z}
\end{aligned}
$$

Further, this result is also valid for all cases of the boundary conditions (19).

\section{Necessary Conditions for PES}

Let us consider

$$
F=\cos \pi z
$$

which obviously satisfies the boundary conditions

$$
F=0 \text { at } z=-1 / 2 \text { and } z=+1 / 2
$$

We shall now consider the boundary conditions (19) in the following forms

$$
\begin{gathered}
w=0=D w \text { at } z= \pm 1 / 2 \\
\text { or } w=0=D^{2} w \text { at } z= \pm 1 / 2 \\
\text { or } w=0=D w \text { and } \\
w=0=D^{2} w \text { at } z= \pm 1 / 2
\end{gathered}
$$

In (25) and (26) the origin has been shifted to the midway for convenience in computation.

Equation (17) upon using (24) yields

$$
\left(D^{2}-a^{2}\right)^{2} w=\cos \pi z
$$

Let $q_{1}^{2}$ and $q_{2}^{2}$ be the roots of the auxiliary equation of the Equation (27), hence the general solution of Equation (27) is given by

$$
w=B_{1} \cosh q_{1} z+B_{2} \cosh q_{2} z+\frac{\cos \pi z}{\left(\pi^{2}+a^{2}\right)^{2}}
$$

Now, using the boundary conditions $w=0 ; \quad D w=0$; and $D^{2} w=0$ at $z= \pm 1 / 2$ in Equation (28), we get following three respective equations as

$$
\begin{gathered}
B_{1} \cosh \frac{q_{1}}{2}+B_{2} \cosh \frac{q_{2}}{2}=0 \\
q_{1} B_{1} \sinh \frac{q_{1}}{2}+q_{2} B_{2} \sinh \frac{q_{2}}{2}= \\
\frac{\pi}{\left(\pi^{2}+a^{2}\right)^{2}}=A(\operatorname{say}) \\
q_{1}^{2} B_{1} \cosh \frac{q_{1}}{2}+q_{2}^{2} B_{2} \cosh \frac{q_{2}}{2}=0
\end{gathered}
$$

Now, solving Equations (29)-(31) for $B_{1}$ and $B_{2}$ for different cases of boundary conditions (26), we get

$$
B_{1}\left\{\begin{array}{l}
=0, \text { for }(26 a) \\
=-\frac{A \cosh \left(q_{2} / 2\right)}{\Delta^{\prime}}, \text { for }(26 b) \\
=-\frac{A q_{2}^{2} \cosh \left(q_{2} / 2\right)}{\Delta}, \text { for }(26 c)
\end{array}\right.
$$


and

$$
B_{2}\left\{\begin{array}{l}
=0, \text { for }(26 a) \\
=-\frac{A \cosh \left(q_{1} / 2\right)}{\Delta^{\prime}}, \text { for }(26 b) \\
=-\frac{A q_{2}^{2} \cosh \left(q_{1} / 2\right)}{\Delta}, \text { for }(26 c)
\end{array}\right.
$$

where,

$$
\Delta=q_{2} \cosh \frac{q_{1}}{2} \sinh \frac{q_{2}}{2}-q_{1} \cosh \frac{q_{2}}{2} \sinh \frac{q_{1}}{2}
$$

and

$$
\Delta^{\prime}=q_{1} q_{2}^{2} \cosh \frac{q_{2}}{2} \sinh \frac{q_{1}}{2}-q_{2} q_{1}^{2} \cosh \frac{q_{1}}{2} \sinh \frac{q_{2}}{2}
$$

Now, substituting the values of $w$ and $F$ in integrals $I_{1}$ and $I_{2}$ and integrating, we get

$$
\begin{gathered}
I_{1}=\int_{-1 / 2}^{+1 / 2}\left\{(D F)^{2}+a^{2}(F)^{2}\right\} d z=\frac{\pi^{2}+a^{2}}{2} \\
I_{2}=\int_{-1 / 2}^{+1 / 2} w F d z \\
=\frac{2 \pi B_{1} \cosh \frac{q_{1}}{2}}{\pi^{2}+q_{1}^{2}}+\frac{2 \pi B_{2} \cosh \frac{q_{2}}{2}}{\pi^{2}+q_{2}^{2}}+\frac{1}{2\left(\pi^{2}+a^{2}\right)^{2}}
\end{gathered}
$$

Using the values of integrals $I_{1}$ and $I_{2}$ given in (34) and (35) in inequality (23), we get

$$
\begin{aligned}
& R_{c} \leq \frac{R_{s}}{\tau}+ \\
& 2 a^{2}\left\{\frac{2 \pi B_{1} \cosh \frac{q_{1}}{2}}{\pi^{2}+q_{1}^{2}}+\frac{\pi^{2}+a^{2}}{\pi^{2}+q_{2}{ }^{2}}+\frac{1}{2\left(\pi^{2}+a^{2}\right)^{2}}\right\}
\end{aligned}
$$

Now, utilizing sufficiently large value of $R_{s}$ in the above inequality (i.e. taking $R_{s} \rightarrow \infty$ ), we have

$$
R_{c} \leq \tau^{-1} R_{s}
$$

which is uniformly valid for all the cases of boundary conditions.

Therefore, combining inequalities (22) and (36), we get

$$
R_{c} \rightarrow \tau^{-1} R_{s}
$$

which is precisely the $\tau^{-1} R_{s}$ - Law for Veronis type thermohaline convection problem.

Further, replacing $R$ and $R_{s}$ by $-|R|$ and $-\left|R_{s}\right|$ respectively, in Equation (8), emulating the proof of $\tau^{-1} R_{s}$ - Law and taking sufficiently large values of $|R|$, we can easily prove the $\tau|R|$ - Law for Stern type thermohaline convection problem also.

\section{Conclusions}

We have studied the thermohaline instability of Veronis and Stern types for the onset of stationary convection for all possible cases of boundary conditions. It is important to point out here that the values of critical Rayleigh number derived by Veronis and Stern for their respective configuration were valid for the idealized case of both dynamically free boundaries. Since, the exact solutions of the eigenvalue problems describing thermal/thermohaline convection are not obtainable in closed form for other two cases (i.e. rigid-rigid and rigid-free) of boundary combinations, therefore the values of critical Rayleigh number for these realistic cases are not known analytically for thermohaline convection problems. However, to obtain the critical Rayleigh number for thermal convection problem for these realistic cases of boundary conditions Chandrasekhar [8] used the numerical computations. In the present analysis the values of critical Rayleigh number for all three cases of boundary conditions have been obtained using the known results of Chandrasekhar [8] for classical Bénard problem. Further, the result obtained by Knobloch [9] for the idealized case of free boundaries also follows from (37).

Veronis [6] derived an asymptotic relation for a configuration which is initially gravitationally stable for the case of both free boundaries. He remarked that for a given very stable salt gradient (so that $R_{s}$ is sufficiently large), $R_{c}$ must have a value that is about 100 times the value of $R_{s}$. In other words, the destabilizing temperature gradient must exceed the stabilizing salt gradient by a factor of 100 . This result clearly violates one's intuition, since it means that the vertical density profile must be highly gravitationally unstable before convection can occur. In Section 3, this asymptotic behaviour of critical Rayleigh number has been extended to the cases of realistic boundary conditions and is validated in Section 5 using the route through variational principle. This is a necessary condition for the validity of PES for Veronis type thermohaline convection and is named as $\tau^{-1} R_{s}$ Law. Analogously, a $\tau|R|$ - Law for Stern type thermohaline convection problem has also been derived here.

\section{Acknowledgements}

Thanks are extended to Professor J. R. Gupta for his perspicacious comments on the subject. One of us (JSD) gratefully acknowledges the financial support of UGC 
under SAP.

\section{References}

[1] H. Bénard, "Les Tourbillons Cellulaires dans une Napple Liquide," Revue Generale des Sciences Pures at Appliqués, Vol. 11, 1900, pp. 1261-1271.

[2] L. Rayleigh, "On the Convective Current in a Horizontal Layer of Fluid When the Higher Temperature is on the Upper Side," Philosophical Magazine, Vol. 32, No. 3, pp. 529-543.

[3] J. S. Turner, "Multicomponent Convection," Annual Review of Fluid Mechanics, Vol. 17, No. 1, 1985, pp. 11-44.

[4] A. Brandt and H. J. S. Fernando, "Double Diffusive Convection," American Geophysical Union, Washington, DC, 1996.
[5] M. E. Stern, "The Salt Fountain and Thermohaline Convection," Tellus, Vol. 12, No. 2, 1960, pp. 172-175.

[6] G. Veronis, "On Finite Amplitude Instability in Thermohaline Convection," Journal of Marine Research, Vol. 23, 1965, pp. 1-17.

[7] J. R. Gupta, J. S. Dhiman and J. Thakur, "Thermohaline Convection of Veronis and Stern Types Revisited," Journal of Mathematical Analysis and Applications, Vol. 264, No. 2, 2001, pp. 398-407.

[8] S. Chandrasekhar, "Hydrodynamic and Hydromagnetic Stability," Oxford University Press, Amen House, London, 1961.

[9] E. Knobloch, "Convection in Binary Fluids," Physics of Fluids, Vol. 23, No. 9, 1980, pp. 1918-1920. 